\title{
Discontinuity Preserving Regularization for Modeling Sliding in Medical Image Registration
}

\author{
Dan Ruan, Student Member, IEEE, Jeffrey A. Fessler, Fellow, IEEE, Selim Esedoḡlu
}

\begin{abstract}
Sliding effects often occur along tissue/organ boundaries. However, most conventional registration techniques either use smooth parametric bases or apply homogeneous smoothness regularization, and fail to address the sliding issue. In this study, we propose a class of discontinuity-preserving regularizers that fit naturally into optimization-based registration. The proposed regularization encourages smooth deformations in most regions, but preserves large discontinuities supported by the data. Variational techniques are used to derive the descending flows. We discuss general conditions on such discontinuity-preserving regularizers, and their properties based on an anisotropic filtering interpretation. Preliminary tests with 2D CT data show promising results.
\end{abstract}

\section{INTRODUCTION}

M EDICAL registration techniques aim to find the coordinate transformation that best matches two images. Organ and tissue motions are complex, and any reasonably accurate description of such motion requires high degrees of freedom. The image registration problems that estimates such nonrigid transformations are known to be ill-posed. As a consequence, prior knowledge about the underlying physical process is incorporated to address this challenge. In particular, smoothness of the transformation is widely utilized: parametric methods use smooth basis functions (such as B-spline) and optimize over relatively small number of coefficients; fully nonparametric methods such as optical flow and biomechanical models with finite elements either build in smoothness constraints or incorporate smoothness regularization in an optimization framework. However, sliding along tissue/organ boundary widely exists, and homogeneous smoothing of the transformation field blurs the estimated transformation across the sliding interface, resulting in undesirable artifacts.

Recently, several studies [1], [2] of joint segmentation and registration have arisen from various disciplines and applications. In these methods, smooth regions and singularity sets (edges) are devised according to image intensity, and registration aims to align each part respectively. The smoothness and discontinuity in the deformation itself is not addressed directly.

Manuscript received November 14, 2008. This work was supported in part by the Barbour scholarship from University of Michigan, US NIH grant P01CA59827, and US NSF grant DMS-0713767. Selim Esedoḡlu acknowledges the support of a Sloan Foundation Fellowship.

Dan Ruan is with the Radiation Oncology Department, Stanford University, Stanford, CA 94305 USA (e-mail: druan@ stanford.edu)

Jeffrey A. Fessler is with the Department of Electrical Engineering and Computer Science, University of Michigan, Ann Arbor, MI 49109 USA (email: fessler@eecs.umich.edu).

Selim Esedoglu is with the Department of Mathematics, University of Michigan, Ann Arbor, MI 48109 USA (e-mail: esedoglu@umich.edu).
In this study, we propose a class of regularization schemes that preserve intrinsic discontinuities in the deformation field itself. We provide general analysis on their functional forms, and some desired properties as a consequence. We derive the descending flow for optimization and discuss briefly some implementation issues. A preliminary 2D test with clinical CT data shows promising results.

\section{BACKGROUND AND EXISTING WORK}

For clarity, we discuss the derivations for 2D case, yet all analysis generalizes naturally to higher dimensions. We represent the deformation vector field $\Phi: \Omega \rightarrow \Re^{2}$ as $\Phi(\boldsymbol{x})=[u(\boldsymbol{x}), v(\boldsymbol{x})]^{T}$, where $\Omega$ indicates the region of interest; $u$ and $v$ are directional deformation and assumed to be orthogonal (but do not have to align with the image coordinate $(x, y))$ in general. Given an source image $f: \tilde{\Omega} \rightarrow \Re$ and a target image $g: \Omega \rightarrow \Re$, the goal of image registration is to find an estimate of the deformation vector field $\hat{\Phi} \in \Gamma$ such

$$
\left.\left.g\right|_{\Omega} \approx f \circ\left(I+\Phi^{*}\right)\right|_{\Omega},
$$

where $\Gamma$ is the feasible set of deformations.

One of the most widely used method is to pose the image registration problem in an optimization framework, and obtain the deformation estimate as the optimal solution to a designed energy functional:

$$
\Phi^{*}=\arg \min _{\Phi \in \Gamma} E(f, g, \Phi) .
$$

Due to the ill-posedness of the image registration problem, the energy $E$ usually consists of two component: a discrepancy term that measures the mismatch between $f \circ(I+\Phi)$ and a regularization term that incorporates prior knowledge about the desired deformation. Mathematically:

$$
E(f . g \Phi)=E_{d}(f \circ(I+\Phi), g)+\lambda E_{r}(f, g ; T),
$$

where $\lambda$ balances the effect of the two competing energies.

The choice of discrepancy measure depends on the data type. In general, sum of squared distance is used for calibrated monomodality registration and mutual information for multi-modality registration. Correlation based metric is also commonly applied.

We focus on regularization energy, and list the most commonly most commonly acknowledged prior knowledge about the deformation and the corresponding mathematical formulation in Table I. 
TABLE I

COMMON PRIOR FOR DEFORMATION $\Phi$

\begin{tabular}{l||l} 
smoothness [3] & $E_{r}=\int\|\nabla \Phi\|^{2}$ \\
topology preservation [4] & $\|\nabla \Phi+I\|>0$ for all $\boldsymbol{x} \in \Omega$ \\
volume preservation [5] & $E_{r}=\int(\|\nabla \Phi+I\|-1)^{2}$ \\
local rigidity [6] & $E_{r}=\int \eta(\boldsymbol{x})\left\|\nabla \Phi \nabla \Phi^{T}-I\right\|$ \\
\hline
\end{tabular}

In this work, we address a different type of prior knowledge: sliding effect along some anatomical structures. It is known that sliding occurs between interior anatomical structures (lung and diaphragm in particular) and the rib-cage during respiratory motion. However, with global smoothness regularization/constraint, the estimated deformation field fails to accurate capture this discontinuity. Some recent studies [1], [2] addressed this problem from the perspective of joint segmentation and registration and demonstrated promising results. However, the underlying assumption that motion discontinuity coincides with intensity discontinuity is questionable. During breathing, there is interaction between the diaphragm and lung expansion/shrinkage. Allowing discontinuity between these two organs would be misleading in estimating the deformation field. In this study, we aim to design a regularization energy that preserves the intrinsic discontinuity of the motion field.

\section{PROPOSED METHOD}

As we are mainly interested in geometric regularization for smoothness/discontinuity, the regularization term is taken to be independent of the image. It corresponds to a special case of the regularized registration problem introduced in (3) with

$$
E_{r}(f, g ; T)=E_{r}(\Phi) \text {. }
$$

A constant weight $\lambda$ is adopted throughout the whole image to balance the data fidelity and regularization energy. We focus on designing $E_{r}$, and assume mono-modality images with $L_{2}$ metric as data fidelity measure hereafter. Thus the goal of registration can be formulated as:

$$
\begin{aligned}
\Phi^{*} & =\arg \min _{\Phi \in \Gamma} E(f, g, \Phi) \\
& =\arg \min _{\Phi \in \Gamma}\left\{\|g-f \circ(I+\Phi)\|_{2}^{2}+\lambda E_{r}(\Phi)\right\} .
\end{aligned}
$$

\section{A. Discontinuity Preserving Regularization}

To encourage smooth deformations in most of the region of interest (ROI), yet admitting some discontinuities requires a "magnitude" measure of the local change of the deformation field, analogous to the norm of image gradient in image restoration. The Jacobian of the deformation $\Phi$ at $\boldsymbol{x}$ is given by:

$$
D \Phi(\boldsymbol{x})=\left[\begin{array}{ll}
u_{x} & u_{y} \\
v_{x} & v_{y}
\end{array}\right] .
$$

We propose to use the Frobenius norm of the matrix $D \Phi(\boldsymbol{x})$ as the local measure of variation for the deformation field:

$$
\begin{aligned}
|D \Phi|_{\text {Frob }} & =\sqrt{u_{x}^{2}+u_{y}^{2}+v_{x}^{2}+v_{y}^{2}} \\
& =\sqrt{|\nabla u|_{2}^{2}+|\nabla v|_{2}^{2}}
\end{aligned}
$$

This matrix norm is independent of both the image coordinate system $(x, y)$ and the deformation vector field direction $(u, v)$. For simplicity, we assume that the $u$ and $v$ components of the vector field correspond to the deformation field in $x$ and $y$ directions respectively hereafter. In addition, this measure of "deformation change" introduces coupling among the various directions in the vector fields and reflects the intuition that we observe a "jump" in the deformation field regardless of the specific direction such change occur, unlike the simple coordinate-wise sum used in traditional optical flow regularization [7], [8]. For simplicity, we make matrix Frobenius norm the default notation for $|D \Phi|$ hereafter and drop the subscript.

We consider a class of regularizers with the form:

$$
E_{r}(\Phi)=\int \phi(|D \Phi|) d \boldsymbol{x} .
$$

Applying variational analysis, and assuming Neumann boundary conditions, i.e., $\partial_{n} u=0$ and $\partial_{n} v=0$ on $\partial \Omega$, we derive the descent flow[7] $w_{r}=\left(u_{r}, v_{r}\right)$ of $E_{r}$ to be as follows:

$$
\begin{aligned}
u_{r} & =\nabla \cdot\left(\frac{\partial}{\partial \nabla u} \phi\right) \\
& =\nabla \cdot\left(\frac{\phi^{\prime}(|D \Phi|)}{|D \Phi|} \nabla u\right) .
\end{aligned}
$$

The expression for the update flow $v_{r}$ for $v$ is similar. For simplicity, we define the "influence function" as $\psi(s) \triangleq \phi^{\prime}(s) / s$.

To design a proper regularization $\phi$ that results in edge preserving flow, we interpret the process as anisotropic filtering and decompose the effect of the flow into the normal and tangent directions for each component of the deformation field. We derive the regularization flow in $u$ - direction as:

$$
\begin{aligned}
u_{r}= & \psi(|D \Phi|)\left(u_{x x}+u_{y y}\right)+\frac{\phi^{\prime \prime}(|D \Phi|)-\psi(|D \Phi|)}{|D \Phi|^{2}} \ldots \\
& \times\left(u_{x}^{2} u_{x x}+2 u_{x} u_{y} u_{x y}+u_{y}^{2} u_{y y}\right) .
\end{aligned}
$$

By convention, we denote the second derivatives of $u$ in the tangent (T-) direction and normal (N-) direction as $u_{T T}$ and $u_{N N}$ respectively, with

$$
\begin{gathered}
u_{T T}=T^{T} \nabla^{2} u T=\frac{1}{|\nabla u|}\left(u_{x}^{2} u_{y y}+u_{y}^{2} u_{x x}-2 u_{x} u_{y} u_{x y}\right) ; \\
u_{N N}=N^{T} \nabla^{2} u N=\frac{1}{|\nabla u|}\left(u_{x}^{2} u_{x x}+u_{y}^{2} u_{y y}+2 u_{x} u_{y} u_{x y}\right) .
\end{gathered}
$$

Rearranging the terms in (9) yields:

$u_{r}=\psi(|D \Phi|) u_{T T}+|\nabla u|^{2}\left(\frac{\phi^{\prime \prime}(|D \Phi|)}{|D \Phi|^{2}}-\frac{\psi(|D \Phi|)}{|D \Phi|^{2}}+\frac{\psi(|D \Phi|)}{|\nabla u|^{2}}\right) u_{N N}$.

For 2D case (higher dimension situations have similar structure):

$$
\frac{\psi(|D \Phi|)}{|\nabla u|^{2}}-\frac{\psi(|D \Phi|)}{|D \Phi|^{2}}=\psi(|D \Phi|) \frac{|\nabla v|^{2}}{|D \Phi|^{2}|\nabla u|^{2}} .
$$

The coupling between $u$ and $v$ in the flow motivates us to consider the contribution of variation in each deformation direction in $|D \Phi|$. We define $\beta_{u} \triangleq \frac{|\nabla u|^{2}}{|D \Phi|^{2}}$ and $\beta_{v} \triangleq \frac{|\nabla v|^{2}}{|D \Phi|^{2}}$. By 
construction, $\beta \in[0,1]$ and $\beta_{u}+\beta_{v}=1$. Then (10) can be rewritten as:

$$
u_{r}=\left(\phi^{\prime \prime}(s) \beta_{u}+\psi(s) \beta_{v}\right) u_{N N}+\psi(s) u_{T T},
$$

Now we are ready to discuss some desired properties for the function $\phi$. This is more complicated than image restoration problems as $\phi$ is intrinsically a function of both $u$ and $v$.

- In the presence of small variations in the deformation, ( $|D \Phi|$ small implies $|\nabla u|,|\nabla v|$ both small), isotropic smoothing is desirable in each individual deformation direction. It is reasonable to require non-trivial smoothing along the tangent direction:

$$
\phi^{\prime}(0)=0, \quad \text { with } \lim _{s \rightarrow 0^{+}} \psi(s)>0 .
$$

To have isotropic diffusion as $s \rightarrow 0^{+}$is equivalent to:

$$
\lim _{s \rightarrow 0^{+}} \beta_{v}+\beta_{u} \frac{\phi^{\prime \prime}(s)}{\psi(s)}=1 .
$$

Together with the fact that $\beta_{u}+\beta_{v}=1$, isotropic diffusion for small deformation implies

$$
\lim _{s \rightarrow 0^{+}} \psi(s)=\lim _{s \rightarrow 0^{+}} \phi^{\prime \prime}(s)>0 .
$$

Once the conditions (12) and (13) are satisfied, the flow (11) for small variation reduces to:

$$
u_{r} \approx \phi^{\prime \prime}(0) \Delta u \text {. }
$$

The same analysis holds for $v_{r}$. We immediately recognize that this diffusion coincides with the isotropic flow from the heat equation.

- In the presence of large variations in deformation (large $|D \Phi|)$, it is desirable to diffuse the deformation along the discontinuity, but not across it. We need to keep in mind that the level of discontinuity $|D \Phi|$ takes into account deformation in all directions, and the diffusion process in a certain direction ( $u$ or $v$ ) is decomposed with respect to its own gradient field. In other words, the diffusion process in $u$ direction is the projection of the joint deformation flow onto that direction. To preserve discontinuity, it suffices to annihilate the coefficients of $u_{N N}$ and $v_{N N}$ for large $|D \Phi|$, and assume non-vanishing coefficients for the tangent flow components.

$$
\left\{\begin{array}{l}
\lim _{s \rightarrow+\infty} \phi^{\prime \prime}(s) \beta_{u}+\psi(s) \beta_{v}=0 ; \\
\lim _{s \rightarrow+\infty} \psi(s)>0 .
\end{array}\right.
$$

If one were to insist on the annihilation of the normal flow for all possible combinations of $\left(\beta_{u}, \beta_{v}\right)$, it would be necessary to require:

$$
\lim _{s \rightarrow+\infty} \phi^{\prime \prime}(s)=0 \text { and } \lim _{s \rightarrow+\infty} \psi(s)=0 .
$$

On the other hand, if $\beta_{u} \approx 0$, indicating that the variation in $x$-direction $(|\nabla u|)$ is relatively small, isotropic diffusion in that direction would not result in over-smoothing discontinuity and should be acceptable. With $v$ being the major contributor to the overall discontinuity in $|D \Phi|$, only $v_{N N}$ has to be annihilated. Unfortunately, this again results in a set of incompatible conditions on $\phi$ :

$$
\lim _{s \rightarrow+\infty} \phi^{\prime \prime}(s) \leq 0 \text { and } \lim _{s \rightarrow+\infty} \psi(s) \geq 0 .
$$

One possible compromise is to let both terms approach zero as $s \rightarrow+\infty$, but at different rates:

$$
\left\{\begin{array}{l}
\lim _{s \rightarrow+\infty} \phi^{\prime \prime}(s)=\lim _{s \rightarrow+\infty} \psi(s)=0 ; \\
\lim _{s \rightarrow+\infty} \frac{\phi^{\prime \prime}(s)}{\psi(s)}=0 .
\end{array}\right.
$$

The above derivation gives a recipe for testing whether a candidate function $\phi$ would induce a discontinuity preserving flow. In fact, many functions satisfy the above conditions (12),(13) and (14), e.g., the hypersurface minimal function $\phi(s)=\sqrt{1+s^{2}}$ [7]. Due to the nonconvex nature of registration problems, we are interested in finding only reasonable local minima in general. In the usual case where $E_{d}$ is nonconvex in $\Phi$, it may be unnecessary to insist on $\phi$ being convex.

We briefly comment on the connection between the general formulation with $\phi$ and some common regularizers here.

- $\phi(s)=s^{2}$ corresponds to the regularization energy:

$$
E_{r, l_{2}}(\Phi)=\int\|\nabla u\|^{2}+\|\nabla v\|^{2} d \boldsymbol{x} .
$$

This is a natural generalization of Tikhonov regularization in image restoration. It is the same energy that Horn and Schunk [3] introduced in the optical flow setting. It is easy to check that $\phi(s)=s^{2}$ violates condition (14), thus fails to preserve discontinuity, which agrees with the known theory.

- $\phi(s)=s$ corresponds to the regularization energy:

$$
E_{r, l_{1}}(\Phi)=\int \sqrt{\|\nabla u\|^{2}+\|\nabla v\|^{2}} d \boldsymbol{x},
$$

which can be regarded as a rotationally invariant generalization of the total variation (TV) regularization for flow fields [9]. Strictly speaking, $\phi(s)=s$ does not satisfy isotropic diffusion condition for small variation, since it has vanished Hessian. Fortunately, almost all numerical implementation of uses a relaxed version by "rounding off" the corner of the $l_{1}$ in a small neighborhood of zero. This relaxation gives $\phi$ quadratic behavior as $s \rightarrow 0^{+}$, making the implemented functional satisfy the required conditions for a discontinuity preserving flow.

\section{A Test Setup with Truncated Quadratic REGULARIZER}

The $L_{2}$ data discrepancy reads,

$$
E_{d}=\frac{1}{2} \int_{\Omega}(g(x)-f(x+\Phi(x)))^{2},
$$

and the corresponding variational descent flow is given by:

$$
w_{\boldsymbol{d}}(\boldsymbol{x})=(g(\boldsymbol{x})-f(\boldsymbol{x}+\boldsymbol{\Phi}(\boldsymbol{x})) \nabla f(\boldsymbol{x}+\boldsymbol{\Phi}(\boldsymbol{x})) .
$$

For the preliminary test, we use a truncated quadratic [10] as the regularization function:

$$
\phi(s, \alpha)= \begin{cases}\left(\frac{\alpha_{0}}{\alpha}\right)^{2} s^{2} & |s| \leq \alpha \\ \alpha_{0}^{2} & \text { otherwise. }\end{cases}
$$

The disadvantage and benefit of this choice are both clear. With strict "saturation" behavior above the scale parameter 


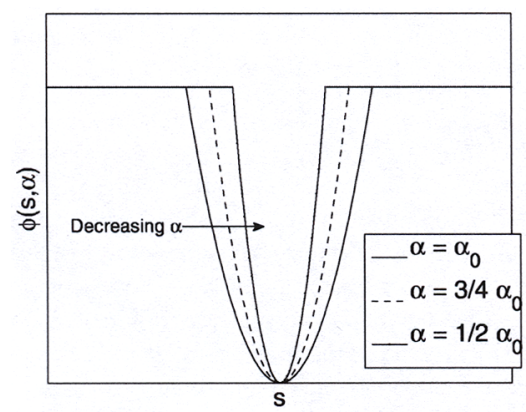

Fig. 1. Truncated quadratic regularization with varying scale.

$\alpha$, it poses a challenge for optimization. Graduated nonconvexification approaches can be utilized. On the other hand, this formulation provides nice theoretical interpretations. It is natural to introduce a line process [11] which is equivalent to "labeling" the outliers in the robust estimation setting [12].

Notice that (17) also provides a simple recipe to extract singularity set $S$ of $|D \Phi|$ from the estimated $\Phi$ by thresholding at level $\alpha$ :

$$
S=\{\boldsymbol{x}:|D \Phi(\boldsymbol{x})|>\alpha\} .
$$

This may be useful for extracting motion interfaces.

To alleviate the local minima issue due to nonconvexity, we start with a large initial $\alpha$ as indicated in Fig. 1. This is equivalent to use the conventional Tikhonov regularization (the vector version is more commonly known as Horn and Schunk in optical flow) of the form $E_{r}=|\nabla u|^{2}+|\nabla v|^{2}$ as $S=\emptyset$ for $\alpha$ large enough. Then the scale parameter $\alpha$ is gradually decreased till the desired tolerance for discontinuity. To speed up the implementation, a multi-resolution scheme is applied.

\section{Preliminary Results}

We applied the proposed method to two coronal CT slices obtained from deep inhale and exhale phases. We chose the tradeoff parameter $\lambda$ 's so that both HS and the truncated quadratic (TQ) regularized results have similar data discrepancy value. Fig. 3 demonstrates that the proposed regularization results in smooth deformation in homogeneous organ region (lung, heart and exterior of rib-cage) and correctly preserves motion interfaces on the motion boundaries. It is known that respiratory motion mainly occur in the superiorinferior (vertical) direction. The depicted vertical deformation components [Fig. 3bottom] illustrate that the proposed method correctly captures the different motions for the interior and exterior of the rib cage. Moreover, it also shows that the interaction between the diaphragm and the lung is the major "driving force" for the respiratory organ motion.

\section{Conclusion}

We have proposed a class of regularizations that preserve discontinuities in deformation fields. It applies a robust estimation function to a coordinate-free measure of deformation variation to ensure smoothness in most ROI, yet also allowing

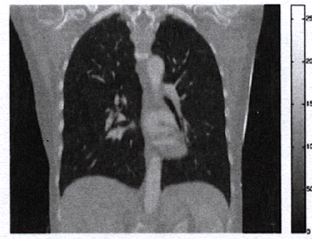

source image (inhale)

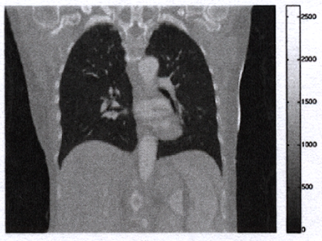

deformed source (HS Reg.)

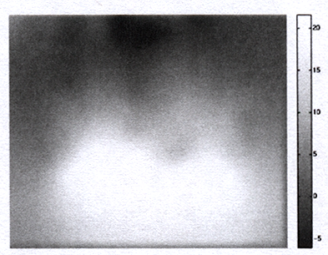

SI def. $U(\boldsymbol{x})$ (HS Reg.)

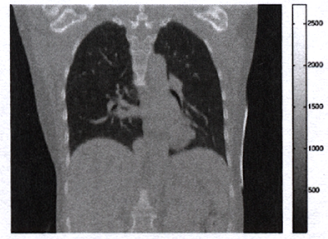

target image (exhale)

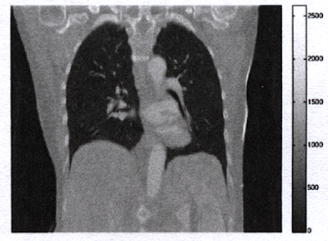

deformed source (TQ Reg.)

SI def. $U(\boldsymbol{x})$ (TQ Reg.)

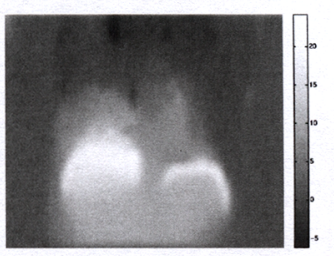

Fig. 2. Registration results with HS and TQ regularizations. Bottom row: superior-inferior deformation component $U$.

for singularities. In the future, we will further investigate numerical aspects of this problem, 3D applications and assess the performance quantitatively.

\section{ACKNOWLEDGMENT}

The authors thank Dr. Marc Kessler for motivating this work and Dr. James Balter for providing the testing data.

\section{REFERENCES}

[1] A. Yezzi, L. Zllei, and T. Kapur, "A variational framework for integrating segmentation and registration through active contours," Med. Im. Anal. vol. 7 , no. 2 , pp. $171-85$, Jun. 2003.

[2] M. Droske and M. Rumpf, "Multiscale joint segmentation and registration of image morphology," IEEE Trans. Patt. Anal. Mech. Intell., vol. 29, no. 12, pp. 2181-2194, Dec. 2007.

[3] B. Horn and B. G. Schunck, "Determining optical flow," Artif. Intell. vol. 18, no. 1-3, pp. 185-203, Aug. 1981.

[4] B. Karacali and C. Davatzikos, "Estimating topology preserving and smooth displacement fields," IEEE Trans. Med. Imag., vol. 23, no. 7 , pp. 868-80, Jul. 2004.

[5] T. Rohlfing, C. R. Maurer, D. A. Bluemke, and M. A. Jacobs, "Volumepreserving nonrigid registration of MR breast images using free-form deformation with an incompressibility constraint," IEEE Trans. Med. Imag., vol. 22, no. 6, pp. 730-741, Jun. 2003.

[6] D. Ruan, J. A. Fessler, M. Roberson, J. Balter, and M. Kessler, "Nonrigid registration using regularization that accommodates local tissue rigidity," in Proc. SPIE 6144, Medical Imaging 2006: Image Proc., 2006, p. 614412.

[7] G. Aubert and P. Kornprobst, Mathmatical Problems in Image Processing, ser. Applied Mathematical Sciences. Springer, 2004.

[8] M. J. Black, G. Sapiro, D. H. Marimont, and D. Heeger, "Robust anisotropic diffusion," IEEE Trans. Im. Proc., vol. 7, no. 3, pp. 421-32, Mar. 1998. 


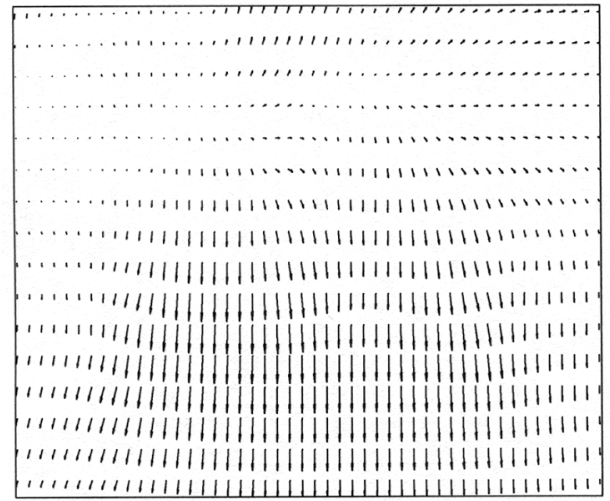

(a) quiver plot of registration result for HS reg.

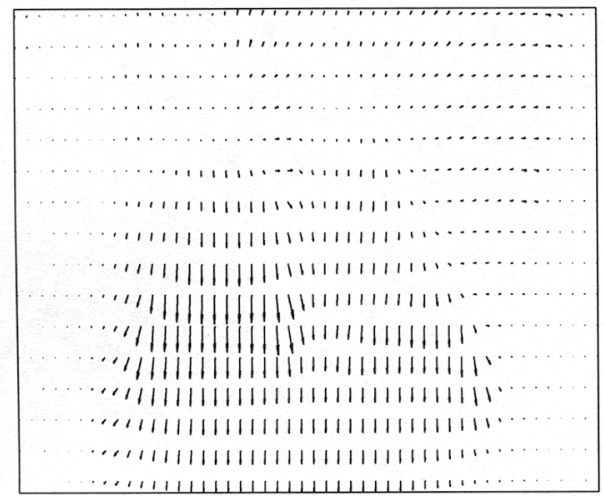

(a) quiver plot of registration result for TQ reg.

Fig. 3. Comparison of deformation fields.

[9] J. Weickert, "On discontinuity-preserving optic flow," in Proc. Computer Vision and Mobile Robotics Workshop, S. Orphanoudakis, P. Trahanias, J. Crowley, and N. Katevas, Eds., Santorini, Sep. 1998, pp. 115-22.

[10] A. Blake and A. Zisserman, Visual Reconstruction. MIT Press, 1987.

[11] S. Geman and D. Geman, "Stochastic relaxation, Gibbs distributions, and Bayesian restoration of images," IEEE Trans. Patt. Anal. Mach. Int., vol. 6, no. 6, pp. 721-41, Nov. 1984.

[12] M. J. Black and A. Rangarajan. "On the unification of line processes, outlier rejection, and robust statistics with applications in early vision," Intl. J. Comp. Vision, vol. 19, no. 1, pp. 57-91, Jul. 1996. 